\title{
Light-triggered release from dye-loaded fluorescent lipid nanocarriers in vitro and in vivo
}

Redouane Bouchaala, ${ }^{\mathrm{a}, \mathrm{b}}$ Nicolas Anton, ${ }^{\mathrm{c}}$ Halina Anton, ${ }^{\mathrm{a}}$ Thierry Vandamme, ${ }^{\mathrm{c}}$ Julien Vermot, ${ }^{\mathrm{d}}$ Djabi Smail, ${ }^{\mathrm{b}}$ Yves Mély, ${ }^{\mathrm{a}}$ Andrey S. Klymchenko,

${ }^{a}$ CNRS UMR 7213, Laboratoire de Biophotonique et Pharmacologie, University of Strasbourg, 74 route du Rhin, 67401 Illkirch Cedex, France

${ }^{b}$ Laboratory of Photonic Systems and Nonlinear Optics, Institute of optics and fine mechanics, University of Setif 1, 19000 Algeria

${ }^{c}$ CNRS UMR 7199, Laboratoire de Conception et Application de Molécules Bioactives, University of Strasbourg, 74 route du Rhin, 67401 Illkirch Cedex, France

${ }^{d}$ IGBMC (Institut de Génétique et de Biologie Moléculaire et Cellulaire), Inserm U964, CNRS

UMR7104, Université de Strasbourg, 1 rue Laurent Fries, 67404 ILLKIRCH, France

*Corresponding author: andrey.klymchenko@unistra.fr

\begin{abstract}
Light is an attractive trigger for release of active molecules from nanocarriers in biological systems. Here, we describe a phenomenon of light-induced release of a fluorescent dye from lipid nano-droplets using visible light. Using auto-emulsification process we prepared nanoemulsion droplets of $32 \mathrm{~nm}$ size encapsulating hydrophobic analogue of Nile Red, NR668. While these nano-droplets cannot spontaneously enter the cells on the time scale of hours, after illumination for 30 seconds under the microscope at the wavelength of NR668 absorption (535 nm), the dye showed fast accumulation inside the cells. The same phenomenon was observed in zebrafish, where nano-droplets initially staining the blood circulation were released into endothelial cells and tissues after illumination. Fluorescence correlation spectroscopy revealed that laser illumination at relatively low power $\left(60 \mathrm{~mW} \mathrm{~cm}^{-2}\right)$ could produce release of the dye into recipient media, such as $10 \%$ serum or blank lipid nanocarriers. The photo-release can be inhibited by deoxygenation with sodium sulfite, suggesting that at least in part the release could be related to photochemical process involving oxygen, though photo-thermal effect could also take place. Finally, we showed that illumination of NR668 can provoke release into the cells of another highly hydrophobic dye co-encapsulated into the lipid nanocarriers. These results suggest dye-loaded lipid nano-droplets as a prospective platform for preparation of light-triggered nanocarriers of active molecules.
\end{abstract}

\section{Keywords:}

Photo-release; controlled release; lipid nanoemulsions; lipophilic dyes; fluorescence correlation spectroscopy; fluorescence microscopy.

Total number of worlds: 6236; Total Number of Figures/Tables: 5. 


\section{Highlights:}

- Light-triggered release from dye-loaded nanoemulsions is shown

- Being stable in vitro and in vivo, nanoemulsion droplets can release their content after illumination with visible (green) light

- Light does not break nano-droplets, but stimulates transfer of their content

- Photo-release of dyes from nanocarriers involves at least in part oxygen photochemistry 


\section{Graphical abstract}

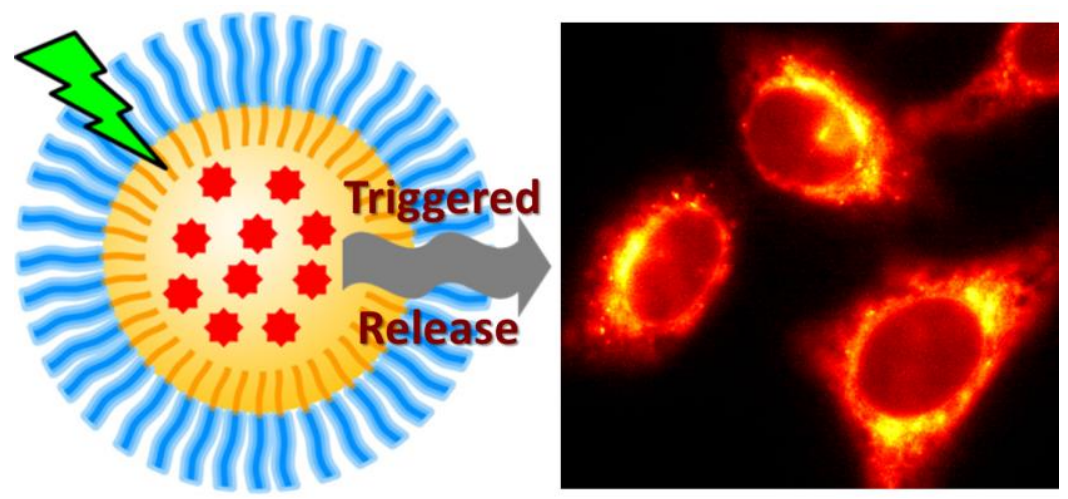

Caption: Lipid nano-droplets loaded with a hydrophobic Nile Red-based fluorescent dye can release their content into cultured cells and zebrafish embryo under illumination with green light. 


\section{Introduction}

Triggered release of active molecules from nanocarriers has been a subject of intensive research in the last decades [1-6]. Various triggers, such as $\mathrm{pH}$, reductive potential, enzymes, temperature, ultrasound, magnetic field and light have been utilized. Light is particularly attractive in this respect, as it can be focused at a desired position of a specimen with high spatiotemporal resolution. The most common approaches for light controlled release utilize molecules that undergo lightcontrolled isomerization, photo-polymerization and photo-cleavage [7, 8]. Thus, azobenzene cistrans isomerization can trigger release from liposomes [9] polymer vesicles and micelles [10, 11] and inorganic porous nanomaterials [12, 13]. Photo cleavage reactions in o-nitrobenzyl [14-16], coumarin [17], spiropyran [18, 19], malachite green [20], cynnamic acid [21, 22] derivatives, etc can produce photo-driven hydrophobicity and charge changes, polymer/lipid fragmentation and de-crosslinking. However, majority of examples of the light-triggered release from nanocarriers reported to date require UV light or two-photon excitation [17, 23]. The other approach utilizes surface plasmon and photo-thermal effects, to trigger the cargo release. In this case, most commonly gold nanoparticles, nano-rods or nano-shells, are used in combination with polymer, lipid nanocarrier or thermo-sensitive covalent bonds [24-28]. The plasmon absorption in this nanostructures was successfully tuned, so that they could be activated with almost any desired excitation wavelength from UV up to near-infrared (NIR) region [29, 30]. However, photothermal effects using gold nanostrcutures generally require relatively strong illumination powers [31], which can be harmful for the biological sample. Upconverting nanoparticles, which convert NIR light into visible and further produce photochemical effect should be also mentioned, but so far all examples of photo-release with upconversion nanoparticles are limited to inorganic systems [32, 33]. In order to develop organic photo-responsive systems one should use dyes encapsulated inside a nanocarrier. In this case, dyes operate as photosensitizers generating highly reactive singlet oxygen and/or as converters of light energy into heat. As photosensitizers, organic dyes, due to their capacity to disrupt biological membranes, were used for photochemical delivery into the cells of molecules and nanoparticles [34-37]. The use of organic dyes as triggers of molecular release from a nanocarrier were mainly realized in lipid vesicles, where local photothermal or photochemical effects were proposed as driving mechanisms of photo-release [38-42]. The photothermal effects were also reported for NIR dyes loaded into nanocarriers in cancer therapy applications [43-45].

Lipid nanoemulsions are an important nanocarrier platform, as they can be readily prepared from FDA approved agents and their hydrophobic core can encapsulate drugs and contrast agents [4650]. Previously, the group of Texier suggested these lipid nanocarriers as imaging agents by loading their core with apolar cyanines $[51,52]$. We further suggested a particular probe design, using long alkyl chains and bulky hydrophobic counterions, that can increase the dye encapsulation together with preservation of the highly efficient fluorescence [53, 54]. Moreover, recent FRETbased studies revealed their high stability in vitro and in vivo and capacity to enter tumors in the intact form $[55,56]$. In particular, we showed that the use of highly hydrophobic substituents of Nile Red enables to significantly increase dye loading, preserve good fluorescence efficiency and 
drastically decrease the dye leakage [53]. However, the question remained: can a strong illumination of a large quantity of dyes confined on the nanoscopic space destabilize lipid droplets? This point was not studied up to now owing to the difficulty to encapsulate a large amount of dyes in these nanoscale reservoirs. Taking recently proposed approaches [53, 54], the dye concentration can be increased up to $5 \mathrm{wt} \%$ along with conserving their fluorescent properties. This is precisely the point that can open the door to photo-triggered properties. Although Nile Red photosensitizing properties were not described yet, we have shown earlier that large concentrations of Nile Redbased membrane probe (NR12S) can produce some photo-damage on cell membranes [57], which suggests that Nile Red as photo-active molecule to destabilize lipid nanocarriers.

In the present work, we describe an unexpected phenomenon, which is a light-triggered release of nano-droplet content, when encapsulating a hydrophobic Nile Red analogue. We show that the dye release can be observed both in cell culture and in vivo on zebrafish model. The light-induced dye release was validated with different acceptor media, such as blank nanocarriers and $10 \%$ serum and it can be inhibited in the presence of oxygen scavenger. Finally, the light-driven release of coencapsulated molecule (second encapsulated dye) along with the Nile Red derivative was validated, which proposes the route to light-controlled drug release from lipid nano-droplets.

\section{Results and Discussion}

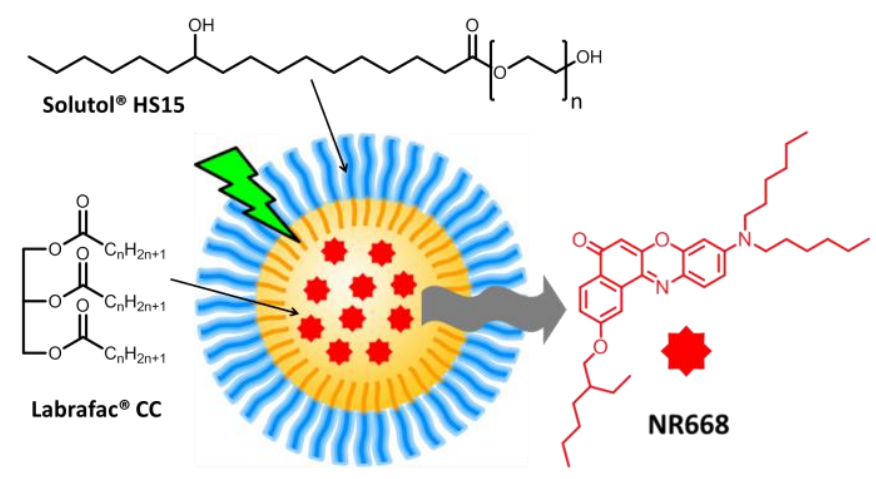

Fig. 1. Concept: lipid nanocarrier and the effect of light on the encapsulated Nile Red derivative NR668.

The idea of light-triggered release from nano-carrier is summarized in Fig. 1. The nano-emulsion droplets can be considered as reservoir with high local concentration of dyes. Our question was whether illumination of this large ensemble of dyes within the small volume of lipid nanocarrier could produce release of its content due to photophysical or photochemical phenomena. As a dye for encapsulation, we selected a derivative of Nile Red bearing long alkyl chains (NR668, Fig. 1). Although this dye is expected to be cell permeant (like parent Nile Red), its high hydrophobicity ensures encapsulation into nano-droplets with minimal leakage effects, which prevents its fast accumulation in the cells [53]. 
We first prepared nanocarriers by spontaneous emulsification of Solutol, Labrafac and NR668 dye in water, which gave $32 \mathrm{~nm}$ particles of low polydispersity index $(<0.1)$ according to dynamic light scattering. The phenomenon of dye release under light illumination was first studied in cell culture. Solutol $^{\circledR}$ (Kolliphor ${ }^{\circledR}$ ) HS 15 / Labrafac ${ }^{\circledR}$ WL nanoemulsions loaded with hydrophobic Nile Red derivative NR668 was incubated with cells and studied by wide-field fluorescence microscopy. Our earlier works showed that no internalization was observed for the same system even after $2 \mathrm{~h}$ of incubation with HeLa cells [53], which showed that these nanocarriers cannot enter the cells on this time scale. Therefore, this system is ideal for testing the effect of light, as no artifacts due to particle internalization or dye leakage into the cells was observed. After $30 \mathrm{~min}$ of incubation of the cells with the emulsion of nanocarriers containing $1 \mathrm{wt} \%$ of NR668, the cells remained poorly fluorescent, as the fluorescence of the medium was more intense than that of the cells. Moreover, after washing of the cells, no significant fluorescence was detected inside the cells (Fig. S1), which confirmed that NR668 nano-droplets did not spontaneously internalize within this time scale. Then, we illuminated the cells surrounded by NR668-loaded nanocarriers for 30 seconds at 535 $\mathrm{nm}$ (absorption maximum of NR668 in nano-droplets is $526 \mathrm{~nm}$ [53]) using the maximal lamp power $\left(\sim 14 \mathrm{~W} / \mathrm{cm}^{2}\right.$ power density at the sample level). The light action resulted in nearly complete photobleaching of the area of observation. Indeed, it was observed that initially very bright fluorescence field became non-fluorescent after the illumination (Fig. 2). Then, with time, the fluorescence of the medium started to recover, but surprisingly the fluorescence of the cells increased with time. This phenomenon was observed already at $1 \mathrm{wt} \%$ of dye loading and took about 10 min to achieve a clear intracellular staining (Fig. 2 A, B), characterized by a significant difference between intracellular and extracellular fluorescence intensity (Fig. 2F). At 5 wt\% dye loading (Fig. 2 C, D), the cell staining after illumination was much stronger, so that the difference between intracellular and extracellular fluorescence intensity was $>3$-fold larger compared to that for $1 \mathrm{wt} \%$ loading. After cell washing from the nanocarriers, we could find the initially illuminated regions, where the fluorescence inside the cells was many-fold larger than that for the nonilluminated regions (Fig. $2 \mathrm{G}, \mathrm{H}$ ). These experiments clearly showed that nanocarriers, unable to enter the cells in the dark, can deliver the dye after illumination. It should be noted that when NR668 dye without lipid nanocarrier was added directly to the cells from its DMSO stock solution (final DMSO concentration was 1\%), the intracellular fluorescence of this dye was observed (Fig. S1). Earlier works showed that its parent analogue Nile Red could also spontaneously internalize [58], which implies that plasma membrane is not really a barrier for these hydrophobic dyes. These results highlight the role of the nanocarrier, which encapsulates NR668 preventing its leakage into the cells, but ensuring its release after the illumination. 


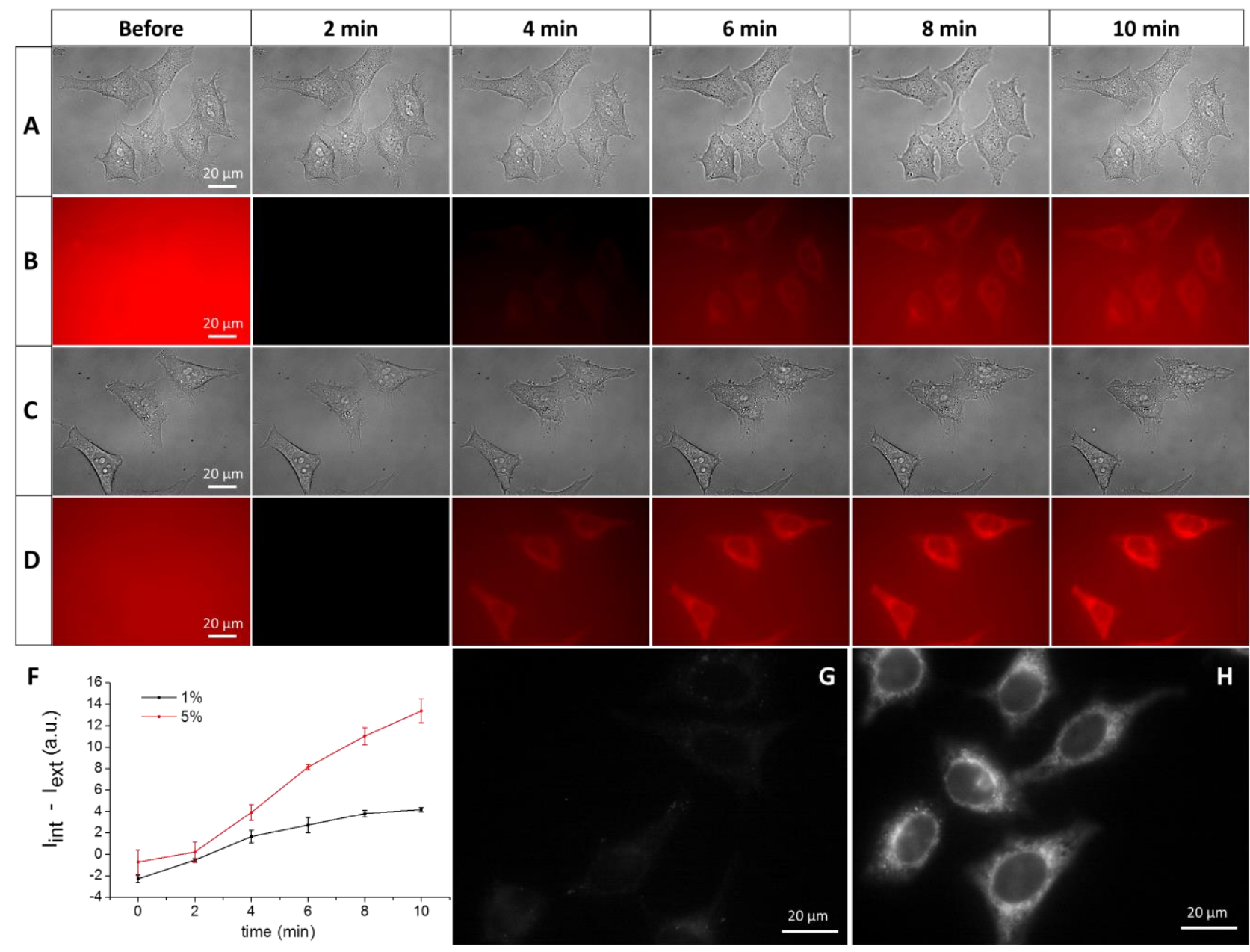

Fig. 2. Release of dye molecules in vitro. Brightfield (A and C) and fluorescence (B and D) images of Hela cells in the presence of nanocarrier loaded with at $1 \%(\mathrm{~A}, \mathrm{~B})$ or $5 \%(\mathrm{C}, \mathrm{D})$ of NR668. The first images on the left correspond to samples before illumination, while all others are after illumination for $30 \mathrm{sec}$ at $535 \mathrm{~nm}$ with 2 min delay for each consecutive image. All the experiments were done at $37^{\circ} \mathrm{C}$. $(\mathrm{F})$ : difference between intracellular and extracellular fluorescence intensity, $I_{\text {int }}-I_{\text {ext }}$, vs time delay after illumination. The error bars represent the standard deviation of the mean from three independent illumination cycles $(n=3)$. (G-H) Fluorescence images after washing (from nanocarriers at $5 \mathrm{wt} \%$ NR668) showing non-illuminated and illuminated regions. 
Control
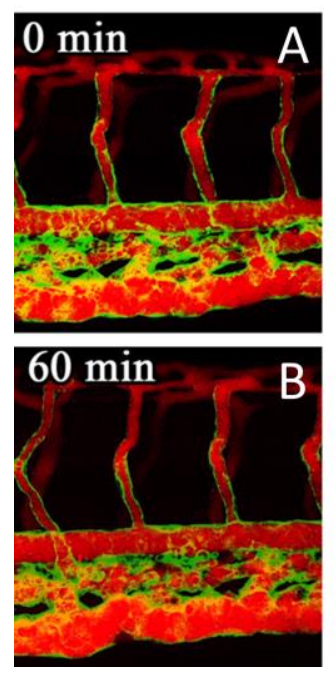

Illuminated
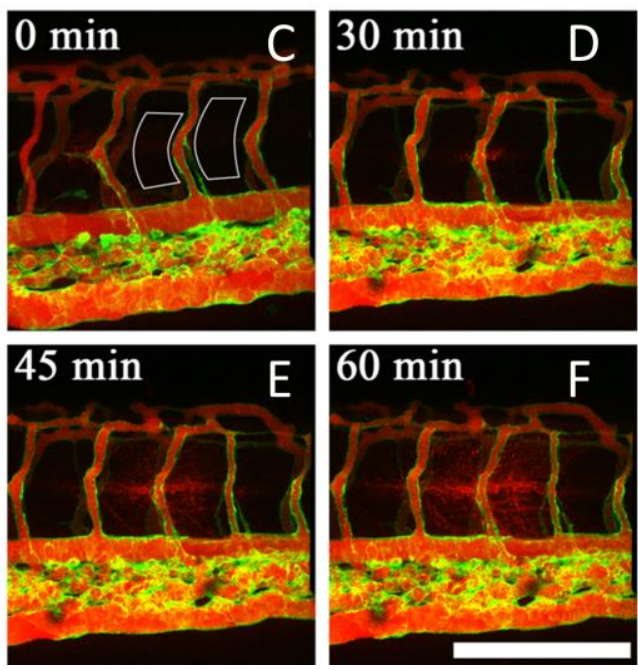

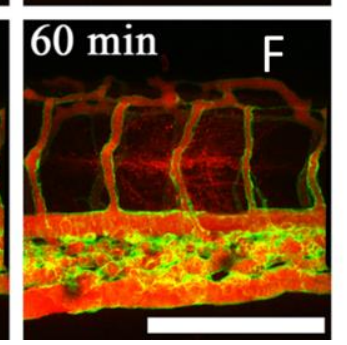

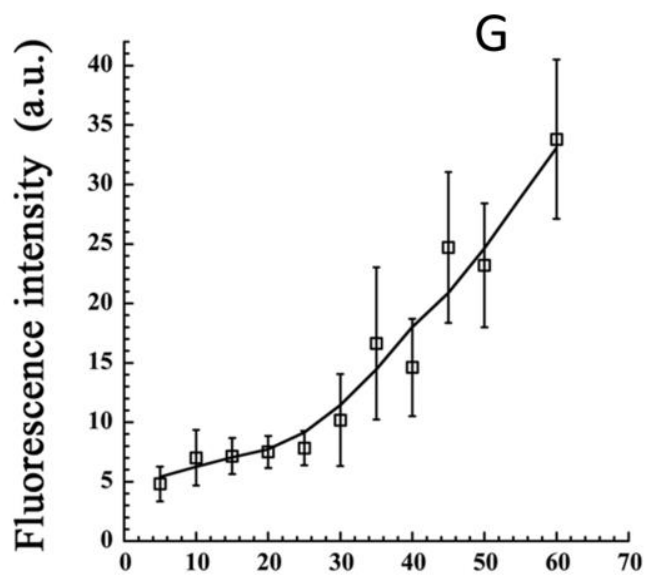

Time after illumination (min)

Fig. 3. The photo-induced release of the NR688 dye from the nanoemulsions in the vasculature of living zebrafish embryo. Fluorescence images of tail vasculature (green) containing NR668 nanoemulsions (red). (A,B) Control non-illuminated embryo, showing no leakage of the dye. (CF) Embryos illuminated for $5 \mathrm{~min}$ through 450-490 $\mathrm{nm}$ bandpass filter, showing a red fluorescence in the tissues surrounding the vessels. Scale bar is $100 \mu \mathrm{m}$. (G) The increase in the fluorescence intensity averaged in the ROIs shown in panel (C) with time. The error bars represent the standard deviation of the mean from two different regions of the embryo $(n=2)$.

Then, we investigated whether the light-induced release can be also realized in vivo on zebrafish. Our earlier work showed that NR668-loaded nanocarriers remained in the blood circulation for at least 30 min without dye leakage into the endothelial cells of the vessels and surrounding tissues [53]. Here, we injected nanocarriers containing $5 \mathrm{wt} \%$ of NR668 and performed microscopy imaging as a function of time without and with illumination (through 450-490 nm bandpass filter). Without illumination, the red fluorescence of nano-droplets was observed exclusively inside the blood vessels for at least $60 \mathrm{~min}$ (Fig 3A,B), indicating that the nanocarriers remain in the blood circulation without dye leakage into the tissues. By contrast, after the illumination, we could observe a progressive increase of the fluorescence in surrounding tissues between the blood vessels (Fig. 3C-F). The fluorescence intensity, recorded in the tissue areas showed continuous increase in time for the observation period of $60 \mathrm{~min}$ (Fig. 3G). Thus, the illumination triggered release of the encapsulated NR668 dye, which then diffused freely into the surrounding tissues. 

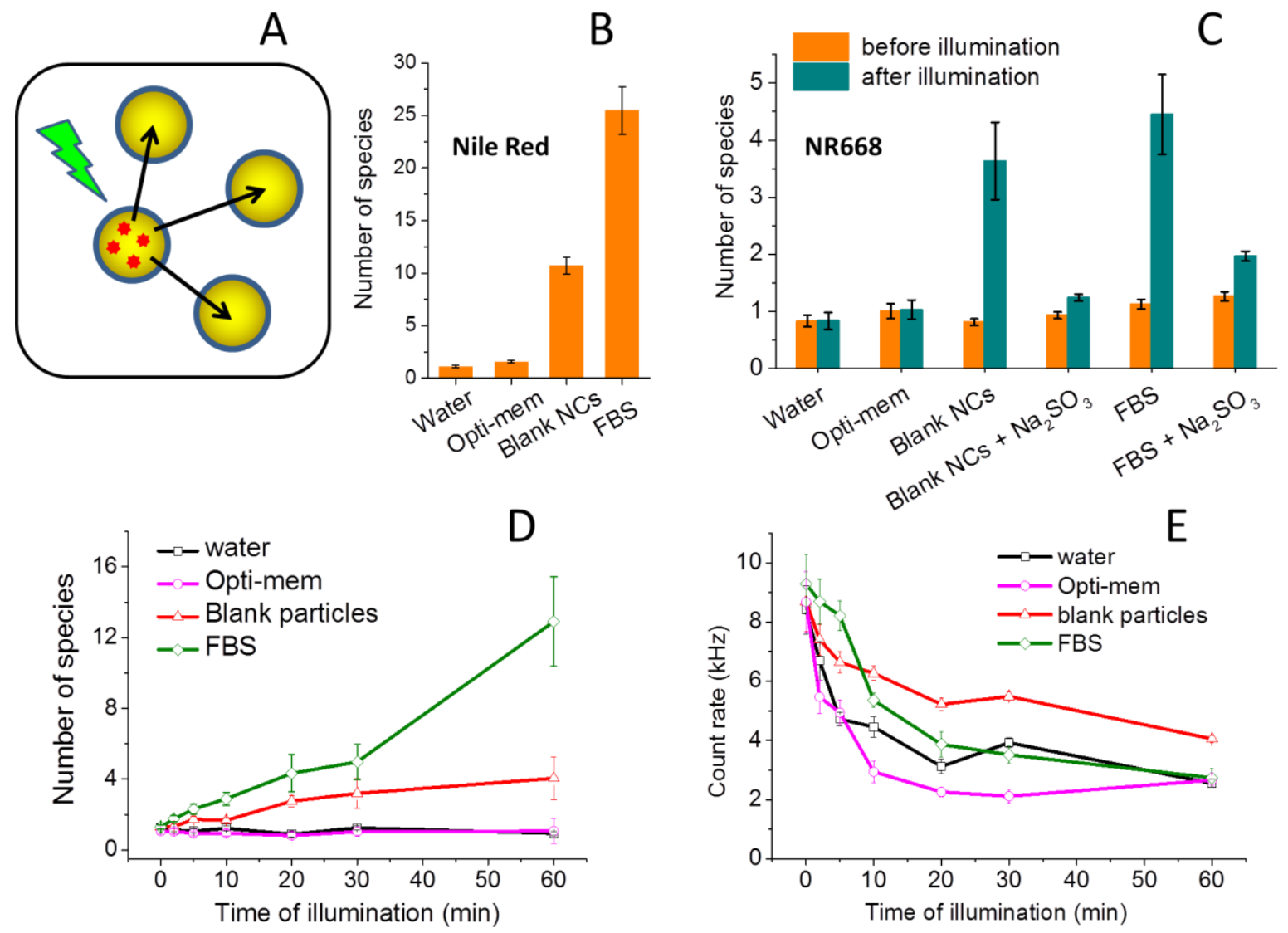

Fig. 4. Fluorescence correlation spectroscopy (FCS) studies of the light-induced dye release into model biological media. (A) Scheme explaining the effect of the dye release on the number of the emissive species. (B) Control experiment showing Nile Red dye release from nanocarriers into blank nanocarriers (NCs) and 10\% serum (FBS), which was recorded as number of emissive species. (C) The effect of $30 \mathrm{~min}$ illumination by $532-\mathrm{nm}$ laser (power density $60 \mathrm{~mW} / \mathrm{cm}^{2}$ ) on lipid nanocarriers loaded with NR668 in different media: water, Opti-MEM, blank nanocarriers and FBS. Presence of oxygen-depleting agent sodium sulfite was also tested. (D) Effect of laser illumination time on the number of emissive species and the total fluorescence intensity of NR668loaded lipid nanocarriers in different media. The error bars in (B-E) represent the standard deviation of the mean ( $n=20$ recorded correlation curves).

To understand better the observed phenomenon of release, we performed fluorescence correlation spectroscopy (FCS) of our nanocarriers in different model biological media. FCS is a powerful technique to characterize nanoparticles in situ, which can provide information about size, concentration and brightness of particles [53, 59, 60]. According to our FCS data, the particle size, measured from the diffusion correlation time of the emissive species was $33 \mathrm{~nm}$ (Table S1). This value is remarkably close to the hydrodynamic diameter obtained for these nanocarriers by DLS $(32 \mathrm{~nm})$. Moreover, the particles concentration for this sample at 1:10000 dilution, evaluated from the number of emissive species per focal volume, was $1.7 \mathrm{nM}$. Basic calculation based on known 
total lipid concentration in the used nanoemulsion $\left(1.1 \times 10^{-3} \%\right)$ and the mass of a single 33-nm nano-droplet $\left(1.9 \times 10^{-17} \mathrm{~g}\right.$, assuming density of the droplet of $\left.1 \mathrm{~g} / \mathrm{ml}\right)$, we could estimate that the concentration of nanocarriers should be around $1.0 \mathrm{nM}$. The correspondence of the theoretical and experimental values of the particle concentration and the match of the particle size obtained from DLS and FCS suggest that after this 10000-fold dilution the nanocarriers remained practically intact.

In order to monitor the photo-release of the dye, we studied the number of emissive species, which, according to our earlier studies, is an important indicator of the dye release [53]. Indeed, if the dyes are released into acceptor medium (blank particles or serum), the number of emissive species should increase (Fig. 4A). For example, in control experiments without illumination, Nile Red encapsulated into the nanocarriers showed low number of emissive species in water and OptiMEM, where poorly soluble Nile Red cannot really escape (Fig. 4B). On the other hand, in the presence of blank nanocarriers or fetal bovine serum (FBS), the number of emissive species increased drastically, showing that Nile Red leaked from nanocarriers and distributed in the recipient medium. By contrast, the number of emissive species for the NR668-loaded nanocarriers was low for all four studied media (Fig. 4C) and the size of emissive species remained stable (Table S1). These results confirmed our earlier data on high stability of NR668-loaded nanocarriers against leakage [53]. After illumination with the laser at $532 \mathrm{~nm}$ (power density $60 \mathrm{~mW} / \mathrm{cm}^{2}$ ) the number of emissive species grew drastically in the presence blank nanocarriers and FBS, but did not change in water and Opti-MEM. The number of emissive species in the media with blank nanocarriers or FBS grew gradually on increase in the time of illumination (Fig. 4D). These results suggest that the dye photo-release is light-dose dependent and it requires biological medium containing hydrophobic microenvironment. This was expected, because NR668 is highly apolar $(\log \mathrm{P}=9.22$ [53]) and practically insoluble in water. Moreover, as the number and the size (Table S1) of emissive species in water did not change after illumination, we could conclude that illumination did not split the nanocarriers into multiple species. Therefore, the observed photorelease in the presence of the acceptor medium as well as in cell and zebrafish embryo is probably related to the light-triggered dye leakage from the nanocarriers into the surrounding hydrophobic microenvironment. The illumination also produced the drop in the total fluorescence intensity (Fig. $4 \mathrm{E}$ ), indicating the photobleaching of the dye. The latter indicates that NR668 underwent photochemical transformation, which could explain the destabilization of the nanocarrier with further release of the dyes into the medium. Photobleaching of dyes is commonly associated with generation of singlet oxygen that further oxidize the dye or other components of the nanocarrier. Therefore, we repeated our FCS experiments in the presence of sodium sulfite. The latter is a reductive deoxygenating agent, which depletes oxygen through oxidation into a sulfate anion [6163]. Remarkably, sodium sulfite drastically decreased the release of the dye under illumination in the presence of blank nanocarriers and serum, as it can be seen from only small change in the number of emissive species (Fig. 4C). Moreover, sodium sulfite did not show a significant influence on the fluorescence intensity of the samples before illumination (Table S1), indicating that it did not act as quencher through electron transfer or other mechanisms. Overall, these results 
suggest that the key mechanism that drives the dye release is linked to the photo-oxidation that could destabilize the nanocarriers.
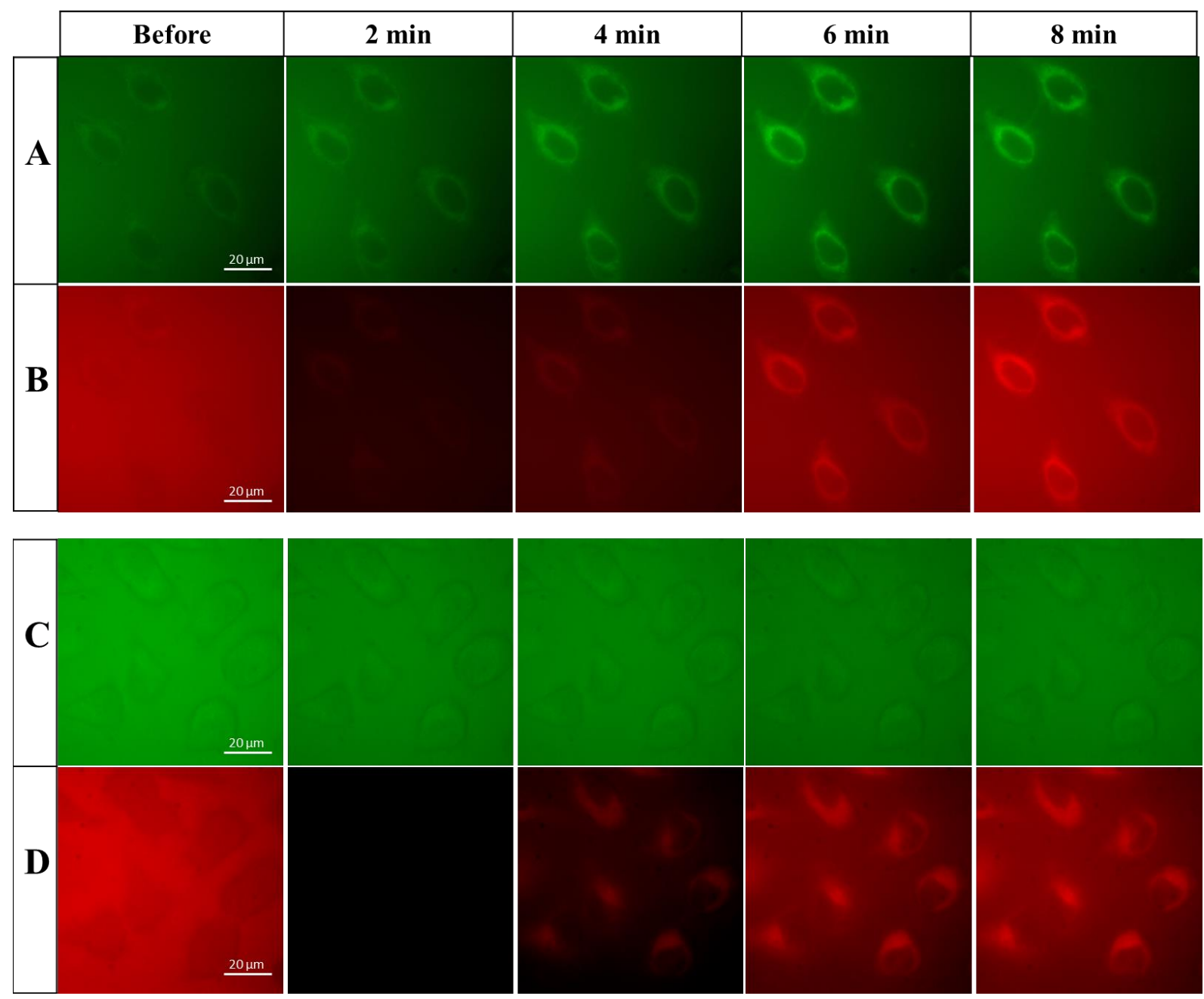

Fig. 5. Fluorescence imaging of HeLa cells incubated for $15 \mathrm{~min}$ at $37{ }^{\circ} \mathrm{C}$ with nanocarrier before and after illumination at $535 \mathrm{~nm}$ for $30 \mathrm{~s}$. Images were taken every $2 \mathrm{~min}$. (A and B) Nanocarriers encapsulating both dyes F888-NR668 (1 wt\% each). (C and G) Mixture of nanocarriers encapsulating separately F888 (1 wt\%) and NR668 (1 wt \%) dyes (1:1, v:v). F888 channel: excitation 360/40nm and emission 470/40nm. NR668 channel: excitation 535/50 nm and emission 610/75 nm.

The important question is whether illumination of NR668 can trigger release of other molecules form the nanocarriers. To this end, we encapsulated into our lipid nano-droplets a second dye, F888, which was also reported previously to remain inside the droplets without dye leakage [53], similarly to NR668. Importantly, the maxima of absorption and emission of F888 in nano-droplets at $1 \mathrm{wt} \%$ loading (388 and $455 \mathrm{~nm}$, respectively) are well separated from those of NR668 (526 and 
$592 \mathrm{~nm}$ ) [53], which should allow selective excitation and detection of three two dyes by fluorescence microscopy. We incubated the dually labeled nanocarriers with cells for $15 \mathrm{~min}$ and then illuminated them for $30 \mathrm{~s}$ at $535 \mathrm{~nm}$. Before illumination, the intracellular fluorescence was weak confirming no leakage of the encapsulated dyes in the presence of cells (Fig. 5 A,B). After illumination, the fluorescence recorded at the NR668 channel showed expected behavior, where the total fluorescence decreased significantly after illumination, and then increased inside the cells in the time period of $10 \mathrm{~min}$ (Fig. 5B). Importantly, the intracellular fluorescence recorded at the channel corresponding to F888 dye (shown in green) increased after illumination and continued increasing together with that of NR668 (Fig. 5A). Importantly, the background fluorescence in F888 channel did not change after illumination, because this illumination cannot excite F888 dye. In the control experiment, we incubated the cells with only F888-loaded nanocarriers. After $30 \mathrm{~s}$ illumination at $535 \mathrm{~nm}$, we found no changes in fluorescence inside the cells (Fig. S2), so that NR668 was absolutely required to induce release of F888 dye. These results showed that illumination that excites selectively NR668 dye inside nano-droplets can trigger the release of the second encapsulated dye F888. Thus, a prototype of a photo-release system is developed, where illumination of nanocarriers could induce the release a molecule of interest, for instance in the drug delivery applications.

Finally, to clarify whether the whole nanocarrier is delivered or just its content, we incubated the cells with a mixture of nano-droplets containing separately NR668 and F888. In this case, if the whole nanocarriers enter after the illumination, then both NR668- and F888-loaded nanocarriers should be detected inside the cells. However, it was found that after illumination, only NR668 signal was observed inside the cells, whereas F888 signal remained low (Fig. 5C,D). This suggested that light illumination triggered release of the dye content from NR668-loaded nanocarrier into the cells, but could not really induce internalization of the whole nanocarrier. Moreover, the fact that F888 did not enter the cells after illumination also suggested that the photorelease process did not disrupt the cell plasma membranes. This feature is important in the development of photo-release systems with minimal photo-damaging effects. Together with the FCS data, these observations suggest that the light acts at the level of the nano-droplet, producing destabilization and further cargo release into the cells. In this respect the described phenomenon is close to the light-triggered release from lipid vesicles, which was explained by photo-thermal or photochemical mechanisms [38-42]. The described phenomenon is also relevant to photochemical internalization [34-37], however, in the latter case light acts mainly at the level of cell membranes. 


\section{Conclusions}

In the present work, a phenomenon of light-induced release from lipid nano-droplets using visible light is described for the first time. It was realized using nanoemulsion droplets of $32 \mathrm{~nm}$ size encapsulating hydrophobic analogue of Nile Red NR668. These nanocarriers do not enter the cells after several hours of incubation at $37{ }^{\circ} \mathrm{C}$, however, after illumination for 30 seconds under the microscope at the wavelength of NR668 absorption $(535 \mathrm{~nm})$, fast accumulation of the released dyes inside the cells was observed. The light-triggered release was also validated in vivo on zebrafish, where nano-droplets showing stable emission in the blood circulation, stained surrounding tissues after illumination. Using fluorescence correlation spectroscopy, we showed that laser illumination at relatively low power $\left(60 \mathrm{~mW} \mathrm{~cm}^{-2}\right)$ produced dye release into $10 \%$ serum or blank lipid nanocarriers. As this process is inhibited by the deoxygenating agent sodium sulfite, we can conclude that the mechanism is linked to oxygen-dependent photochemistry, which destabilizes the nanocarrier. Nevertheless, we cannot exclude that photo-thermal effect could also take part, as proposed in some recent literature for NIR dyes. Finally, we showed that illumination of NR668 can induce release of another highly hydrophobic dye from the lipid nanocarriers, which was observed as accumulation of both dyes inside living cells. Based on these results, dye-loaded lipid nano-droplets emerge as a promising platform for light-triggered release of active molecules in biomedical applications. We expect that this concept could be readily extended to other dyes, which could induce under light stimulus the release drugs from nano-droplets.

\section{Materials and methods}

\section{Materials}

All chemicals and solvents were purchased from Sigma-Aldrich. Kolliphor ${ }^{\circledR}$ HS15 non-ionic surfactant (mixture of polyethylene glycol 660 hydroxystearate and free polyethylene glycol 660 and) obtained from BASF (Ludwigshafen, Germany) was a kind gift from Laserson (Etampes, France), Labrafac ${ }^{\circledR}$ WL (medium chain triglycerides) was obtained from Gattefossé (Saint-Priest, France). MilliQ-water (Millipore) was used in all experiments. Culture reagents were obtained from Sigma (St. Louis, USA), Lonza (Verviers, Belgium) and Gibco-Invitrogen (Grand Island, USA). Opti-MEM ${ }^{\circledR}$ reduced serum medium was from Gibco. Dihexylamino-2-(2-ethyl-hexyloxy)benzo[a]phenoxazin-5- one (NR668) and 4'-Dioctylamino-3-octyloxyflavone (F888) were synthesized as described before [53].

\section{Formulation and characterization of lipid nanoparticle}

Lipid nanocarriers were prepared by spontaneous nano-emulsification. Briefly, NR668 or F888 was solubilised in Labrafac ${ }^{\circledR}$ WL. Then, Kolliphor ${ }^{\circledR}$ HS 15 was added and the mixture was 
homogenized under magnetic stirring at $90^{\circ} \mathrm{C}$. Nano-droplets of $32 \mathrm{~nm}$ diameter were prepared by adding $230 \mathrm{mg}$ of ultrapure water to a mixture of $35 \mathrm{mg}$ of Labrafac ${ }^{\circledR} \mathrm{WL}$ and $65 \mathrm{mg}$ of Kolliphor ${ }^{\circledR}$ HS 15 under intense stirring.

The size distribution of the nanoemulsions was determined by dynamic light scattering on a Zetasizer ${ }^{\circledR}$ Nano series DTS 1060 (Malvern Instruments S.A., Worcestershire, UK) and by FCS (home-built setup, see below). In the DLS measurements statistics by volume was used.

\section{Cell culture}

HeLa cells were cultured in Dulbecco's modified Eagle medium (D-MEM, high glucose, Gibcoinvitrogen) supplemented with $10 \%$ (v/v) fetal bovine serum (FBS, Lonza), $1 \%$ antibiotic solution (penicillin-streptomycin, Gibco-invitrogen) in a humidified incubator with $5 \% \mathrm{CO}_{2}$ at $37^{\circ} \mathrm{C}$. Cells plated on a $75 \mathrm{~cm}^{2}$ flask at a density of $10^{6}$ cells/flask were harvested at $80 \%$ confluence with trypsin-EDTA (Sigma) and seeded onto a chambered coverglass (IBiDi) at a density of $0.1 \times 10^{6}$ cells/IBiDi. After $24 \mathrm{~h}$, cells in the IBiDi dishes were washed 2 times with PBS (phosphate buffer saline) (Lonza). Then, a solution of dye-loaded nano-droplets diluted at 1:1000 in Opti-MEM was added. Microscopy images were taken after 30 -min incubation at $37{ }^{\circ} \mathrm{C}$, unless indicated.

\section{Microscopy}

Fluorescence images were taken on a Leica DMIRE2 inverted microscope equipped with a Leica DC350FX CCD camera and a thermostated chamber (Life Imaging Services, Basel Switzerland) for maintaining the temperature at $37^{\circ} \mathrm{C}$. Cy3 filter cube (excitation $535 / 50 \mathrm{~nm}$, emission 610/75 $\mathrm{nm}$ ) and DAPI filter cube (excitation 360/40nm, emission 470/40nm) were used for detection of NR668 and F888, respectively. A 63× HCX PLAPO (1.32 NA) was used as an objective. In the photo-release studies, the illumination for $30 \mathrm{~s}$ was done using the same Cy3 filter cube at $\sim 14$ $\mathrm{W} / \mathrm{cm}^{2}$ power density of light.

\section{Fluorescence correlation spectroscopy (FCS) and dye release}

FCS measurements were performed on a two-photon platform including an Olympus IX70 inverted microscope. Two-photon excitation at $830 \mathrm{~nm}(10 \mathrm{~mW}$ laser output power) was provided by a mode-locked Tsunami Ti : sapphire laser pumped by a Millenia V solid state laser (Spectra Physics). The measurements were realized in an 96 well plate, using a $50 \mathrm{uL}$ volume per well. The focal spot was set about $20 \mu \mathrm{m}$ above the plate. The normalized autocorrelation function, $\mathrm{G}(\mathrm{t})$ was calculated online by an ALV-5000E correlator (ALV, Germany) from the fluorescence fluctuations, $\delta \mathrm{F}(\mathrm{t})$, by $\mathrm{G}(\tau)=\left\langle\delta \mathrm{F}(\mathrm{t}) \delta \mathrm{F}(\mathrm{t}+\tau)>/<\mathrm{F}(\mathrm{t})>^{2}\right.$ where $\langle\mathrm{F}(\mathrm{t})>$ is the mean fluorescence signal, and $\tau$ is the lag time. Assuming that lipid nanoparticle diffuse freely in a Gaussian excitation 
volume, the correlation function, $\mathrm{G}(\tau)$, calculated from the fluorescence fluctuations was fitted according to Thompson [64]:

$G(\tau)=\frac{1}{N}\left(1+\frac{\tau}{\tau_{d}}\right)^{-1}\left(1+\frac{1}{s^{2}} \frac{\tau}{\tau_{d}}\right)^{\frac{-1}{2}}$

where $\tau_{\mathrm{d}}$ is the diffusion (correlation) time, $\mathrm{N}$ is the mean number of fluorescent species within the two-photon excitation volume, and $\mathrm{S}$ is the ratio between the axial and lateral radii of the excitation volume. The excitation volume is about $0.34 \mathrm{fL}$ and $\mathrm{S}$ is about 3 to 4 . Typical data recording times were $5 \mathrm{~min}$, using dye-loaded lipid nanoparticle diluted 1:10 000 from the originally prepared nanoparticle. Using 6-carboxytetramethylrhodamine (TMR from Sigma-Aldrich) in water as a reference, the hydrodynamic diameter, $d$, of nanocarriers (NCs) was calculated as: $d_{\mathrm{NCs}}=\tau_{\mathrm{d}(\mathrm{NCs})}$ / $\tau_{\mathrm{d}(\mathrm{TMR})} \times \mathrm{d}_{\mathrm{TMR}}$, where $\mathrm{d}_{\text {TMR }}$ is a hydrodynamic diameter of TMR $(1.0 \mathrm{~nm})$. Concentration of NCs was calculated from the number of species by: $\mathrm{C}_{\mathrm{NCs}}=\mathrm{N}_{\mathrm{NCs}} / \mathrm{N}_{\mathrm{TMR}} \times \mathrm{C}_{\mathrm{TMR}}$, using a TMR concentration of $50 \mathrm{nM}$. The data were obtained based on 20 recorded correlation curves; the recording time for each curve was $10 \mathrm{~s}$ for NCs and $30 \mathrm{~s}$ for TMR.

For the dye release studies by FCS, dye-loaded nano-droplets were diluted 10000 fold to four different media: water, Opti-mem, blank nanocarriers at 1000-fold dilution (10-fold excess with respect to dye-loaded droplets) and $10 \%$ of FBS in water (serum). Each sample was placed into special $50 \mu \mathrm{l}$ cuvette and the whole sample was illuminated with $532-\mathrm{nm}$ laser at $\sim 60 \mathrm{~mW} / \mathrm{cm}^{2}$ power density for different time (10 and $30 \mathrm{~s}, 10,2030$ and $60 \mathrm{~min}$ ) and we measure number of species and total count rate.

\section{In vivo studies in zebrafish}

Zebrafish were kept at $28{ }^{\circ} \mathrm{C}$ and bred under standard conditions. The transgenic line, $\operatorname{Tg}$ (flk1:eGFP) [65], expressing eGFP specifically in the endothelial cells, was used in order to visualize the vasculature. For the angiography, the embryos, 3 days after fertilization, were anaesthetized in egg water containing $0.04 \%$ tricaine and $0.05 \%$ phenyl thiourea (Sigma-Aldrich) and immobilized in $0.8 \%$ low melting point agarose (Sigma). The injections were performed using a Nanoject microinjector (Drummond Scientific, Broomal, PA, USA). The glass capillary was filled with NR668-loaded nanoparticle (5 wt\% in oil) of the nanoparticle solution in $5 \mathrm{mM}$ HEPES and $2.3 \mathrm{~nL}$ were injected in the sinus venosus of the embryos. The nanoparticle was immediately distributed in all the vasculature. The injected embryos were placed on the microscope stage and imaged within 5 min after injection. Intravital confocal microscopy was performed on a Leica SP5 fixed stage direct microscope with a $25 \times$ (NA 0.95) and 10 $\times$ (NA 0.3) water immersion objectives. $488 \mathrm{~nm}$ argon laser line was used to excite both eGFP and the NR668 dyes, and their emission was detected by two separate PMTs in the spectral range 500-530 nm and 620-650 nm, respectively. 
At these conditions, no cross-talk between the channels was observed. The illuminations for the light-driven release were performed with the in epi fluorescence mode using a 450-490 nm band pass filter. Confocal z-stacks and time lapses were recorded and treated by the ImageJ software (rsbweb.nih.gov/ij/). The embryos were imaged during one hour post injection and no toxicity due to the injection or to the illumination was observed. All experiments performed with zebrafish complied with the European directive 86/609/CEE and IGBMC guidelines validated by the regional committee of ethics.

\section{Acknowledgements}

This work was supported by ERC Consolidator grant BrightSens 648528. R. Bouchaala was supported by Ministry of Higher Education and Scientific Research of Algeria. We thank Ievgen Shulov for re-synthesizing NR668, L. Richert, P. Didier and F. Przybilla for help with FCS, we thank R. Vauchelles from PIQ imaging platform for help with fluorescence imaging. We also acknowledge help from the IGBMC imaging and zebrafish facilities.

\section{References}

[1] E. Fleige, M.a. Quadir, R. Haag, Advanced drug delivery reviews, 64 (2012) 866-884.

[2] S. Kaur, C. Prasad, B. Balakrishnan, R. Banerjee, Biomaterials Science, 3 (2015) 955-987.

[3] V.P. Torchilin, Nature Reviews Drug Discovery, 13 (2014) 813-827.

[4] S. Mura, J. Nicolas, P. Couvreur, Nat. Mater., 12 (2013) 991-1003.

[5] J. Liu, C. Detrembleur, S. Mornet, C. Jerome, E. Duguet, Journal of Materials Chemistry B, 3 (2015) 6117-6147.

[6] S. Swaminathan, J. Garcia-Amoros, A. Fraix, N. Kandoth, S. Sortino, F.M. Raymo, Chem. Soc. Rev., 43 (2014) 4167-4178.

[7] N. Fomina, J. Sankaranarayanan, A. Almutairi, Advanced drug delivery reviews, 64 (2012) 1005-1020.

[8] J. Olejniczak, C.-J. Carling, A. Almutairi, Journal of Controlled Release, 219 (2015) 18-30.

[9] K. Kano, Y. Tanaka, T. Ogawa, M. Shimomura, Y. Okahata, T. Kunitake, Chem. Lett., (1980) 421-424.

[10] Y. Wang, P. Han, H. Xu, Z. Wang, X. Zhang, A.V. Kabanov, Langmuir, 26 (2010) 709-715.

[11] G. Wang, X. Tong, Y. Zhao, Macromolecules, 37 (2004) 8911-8917.

[12] S. Angelos, E. Choi, F. Vo, L.D. Cola, J.I. Zink, P. Institut, • J. Phys. Chem. C, 111 (2007) 6589-6592.

[13] Y. Tian, Y. Kong, X.J. Li, J. Wu, A.C.T. Ko, M. Xing, Colloid Surf. B-Biointerfaces, 134 (2015) 147-155.

[14] J.Q. Jiang, X. Tong, D. Morris, Y. Zhao, Macromolecules, 39 (2006) 4633-4640.

[15] N. Fomina, C. McFearin, M. Sermsakdi, O. Edigin, A. Almutairi, J. Am. Chem. Soc., 132 (2010) 95409542.

[16] X. Zhao, M. Qi, S. Liang, K. Tian, T. Zhou, X. Jia, J. Li, P. Liu, Acs Applied Materials \& Interfaces, 8 (2016) 22127-22134.

[17] J. Babin, M. Pelletier, M. Lepage, J.-F. Allard, D. Morris, Y. Zhao, Angewandte Chemie-International Edition, 48 (2009) 3329-3332.

[18] H.-i. Lee, W. Wu, J.K. Oh, L. Mueller, G. Sherwood, L. Peteanu, T. Kowalewski, K. Matyjaszewski, Angewandte Chemie-International Edition, 46 (2007) 2453-2457. 
[19] Q.J. Xing, N.J. Li, D.Y. Chen, W.W. Sha, Y. Jiao, X.X. Qi, Q.F. Xu, J.M. Lu, Journal of Materials Chemistry B, 2 (2014) 1182-1189.

[20] R.M. Uda, Y. Kato, M. Takei, Colloid Surf. B-Biointerfaces, 146 (2016) 716-721.

[21] M. Wang, J.-C. Kim, International journal of pharmaceutics, 468 (2014) 243-249.

[22] D. Shi, M. Matsusaki, M. Akashi, Journal of controlled release : official journal of the Controlled Release Society, 149 (2011) 182-189.

[23] N. Fomina, C.L. McFearin, M. Sermsakdi, J.M. Morachis, A. Almutairi, Macromolecules, 44 (2011) 8590-8597.

[24] L. Dykman, N. Khlebtsov, Chemical Society Reviews, 41 (2012) 2256-2282.

[25] A.B.S. Bakhtiari, D. Hsiao, G. Jin, B.D. Gates, N.R. Branda, Angewandte Chemie (International ed. in English), 48 (2009) 4166-4169.

[26] Y. Cheng, T.L. Doane, C.-H. Chuang, A. Ziady, C. Burda, Small (Weinheim an der Bergstrasse, Germany), 10 (2014) 1799-1804.

[27] L. Paasonen, T. Laaksonen, C. Johans, M. Yliperttula, K. Kontturi, A. Urtti, Journal of controlled release : official journal of the Controlled Release Society, 122 (2007) 86-93.

[28] W.-C. Jeong, S.-H. Kim, S.-M. Yang, Acs Applied Materials \& Interfaces, 6 (2014) 826-832.

[29] X.H. Huang, I.H. El-Sayed, W. Qian, M.A. El-Sayed, J. Am. Chem. Soc., 128 (2006) 2115-2120.

[30] E. Ju, Z. Li, Z. Liu, J. Ren, X. Qu, Acs Applied Materials \& Interfaces, 6 (2014) 4364-4370.

[31] Z. Qin, J.C. Bischof, Chemical Society Reviews, 41 (2012) 1191-1217.

[32] B. Yan, J.-C. Boyer, D. Habault, N.R. Branda, Y. Zhao, J. Am. Chem. Soc., 134 (2012) 16558-16561.

[33] Y. Yang, F. Liu, X. Liu, B. Xing, Nanoscale, 5 (2013) 231-238.

[34] H.L. Lu, W.J. Syu, N. Nishiyama, K. Kataoka, P.S. Lai, Journal of Controlled Release, 155 (2011) 458464.

[35] P.K. Selbo, A. Weyergang, A. Høgset, O.J. Norum, M.B. Berstad, M. Vikdal, K. Berg, Journal of Controlled Release, 148 (2010) 2-12.

[36] S. Febvay, D.M. Marini, A.M. Belcher, D.E. Clapham, Nano Letters, 10 (2010) 2211-2219.

[37] A. Høgset, L. Prasmickaite, P.K. Selbo, M. Hellum, B. Ø. Engesæter, A. Bonsted, K. Berg, Advanced Drug Delivery Reviews, 56 (2004) 95-115.

[38] V.C. Anderson, D.H. Thompson, Biochimica Et Biophysica Acta, 1109 (1992) 33-42.

[39] S.J. Leung, M. Romanowski, Theranostics, 2 (2012) 1020-1036.

[40] S. Guha, S.K. Shaw, G.T. Spence, F.M. Roland, B.D. Smith, Langmuir, 31 (2015) 7826-7834.

[41] K.A. Dendramis, D.T. Chiu, J. Am. Chem. Soc., 131 (2009) 16771-16778.

[42] A. Rodriguez-Pulido, A.I. Kondrachuk, D.K. Prusty, J. Gao, M.A. Loi, A. Herrmann, Angewandte ChemieInternational Edition, 52 (2013) 1008-1012.

[43] J. Yu, D. Javier, M.A. Yaseen, N. Nitin, R. Richards-Kortum, B. Anvari, M.S. Wong, J. Am. Chem. Soc., 132 (2010) 1929-1938.

[44] G.T. Spence, G.V. Hartland, B.D. Smith, Chemical Science, 4 (2013) 4240-4244.

[45] G. Chen, K. Wang, Y. Zhou, L. Ding, A. Ullah, Q. Hu, M. Sun, D. Oupický, Acs Applied Materials \& Interfaces, 8 (2016) 25087-25095.

[46] D.J. McClements, Y. Li, Advances in Colloid and Interface Science, 159 (2010) 213-228.

[47] M.M. Fryd, T.G. Mason, Ann. Rev. Phys. Chem., 63 (2012) 493-518.

[48] D.J. McClements, Emulsion Design to Improve the Delivery of Functional Lipophilic Components, in: M.P. Doyle, T.R. Klaenhammer (Eds.) Annual Review of Food Science and Technology, Vol 1, vol. 1, 2010, pp. 241-269.

[49] A. Gianella, P.A. Jarzyna, V. Mani, S. Ramachandran, C. Calcagno, J. Tang, B. Kann, W.J.R. Dijk, V.L. Thijssen, A.W. Griffioen, G. Storm, Z.A. Fayad, W.J.M. Mulder, ACS Nano, 5 (2011) 4422-4433.

[50] N. Anton, J.-P. Benoit, P. Saulnier, Journal of Controlled Release, 128 (2008) 185-199. 
[51] M. Goutayer, S. Dufort, V. Josserand, A. Royere, E. Heinrich, F. Vinet, J. Bibette, J.L. Coll, I. Texier, Eur. J. Pharm. Biopharm., 75 (2010) 137.

[52] I. Texier, M. Goutayer, A.D. Silva, L. Guyon, N. Djaker, I. Veronique Josserand, E. Neumann, J. Bibette, F. Vinet, J. Biomed. Opt., 14 (2009).

[53] A.S. Klymchenko, E. Roger, N. Anton, H. Anton, I. Shulov, J. Vermot, Y. Mely, T.F. Vandamme, RSC Adv., 2 (2012) 11876-11886.

[54] V.N. Kilin, H. Anton, N. Anton, E. Steed, J. Vermot, T.E. Vandamme, Y. Mely, A.S. Klymchenko, Biomaterials, 35 (2014) 4950-4957.

[55] J. Gravier, L. Sancey, S. Hirsjaervi, E. Rustique, C. Passirani, J.-P. Benoit, J.-L. Coll, I. Texier, Molecular Pharmaceutics, 11 (2014) 3133-3144.

[56] R. Bouchaala, L. Mercier, B. Andreiuk, Y. Mély, T. Vandamme, N. Anton, J.G. Goetz, A.S. Klymchenko, Journal of Controlled Release, 236 (2016) 57-67.

[57] Z. Darwich, A.S. Klymchenko, D. Dujardin, Y. Mely, RSC Advances, 4 (2014) 8481-8488.

[58] O.A. Kucherak, S. Oncul, Z. Darwich, D.A. Yushchenko, Y. Arntz, P. Didier, Y. Mely, A.S. Klymchenko, J. Am. Chem. Soc., 132 (2010) 4907.

[59] E. Haustein, P. Schwille, Fluorescence correlation spectroscopy: Novel variations of an established technique, in: Annu. Rev. Biophys. Biomol. Struct., vol. 36, 2007, pp. 151-169.

[60] D. Woll, Rsc Advances, 4 (2014) 2447-2465.

[61] M.E.D. Garcia, A. Sanzmedel, Anal. Chem., 58 (1986) 1436-1440.

[62] M.A. Filatov, S. Baluschev, K. Landfester, Chemical Society Reviews, 45 (2016) 4668-4689.

[63] S.H.C. Askes, N.L. Mora, R. Harkes, R.I. Koning, B. Koster, T. Schmidt, A. Kros, S. Bonnet, Chemical Communications, 51 (2015) 9137-9140.

[64] N.L. Thompson, in: J.R. Lakowicz (Ed.) Topics in Fluorescence Spectroscopy Techniques, vol. 1, Plenum Press, New York, 1991, pp. 337.

[65] S.-W. Jin, D. Beis, T. Mitchell, J.-N. Chen, D.Y.R. Stainier, Development (Cambridge, England), 132 (2005) 5199-5209. 